\title{
POTENCIAIS EVOCADOS AUDITIVOS POR FREQUÊNCIA ESPECÍFICA EM LACTENTES COM AUDIÇÃO NORMAL
}

\author{
Frequency-specific Auditory Brainstem Response in infants \\ with normal hearing Auditory evoked potentials
}

Mabel Gonçalves Almeida ${ }^{(1)}$, Gabriela Ribeiro Ivo Rodrigues (2), Doris Ruthy Lewis ${ }^{(3)}$

\begin{abstract}
RESUMO
Objetivo: determinar os níveis mínimos de resposta (NMR) e a latência da onda $V$ em lactentes ouvintes nas frequências de 0.5, 1, 2 e $4 \mathrm{kHz}$. Método: foram avaliados 23 lactentes sem indicadores de risco para deficiência auditiva que apresentavam emissões otoacústicas evocadas por estímulo transiente (EOAET) e potenciais evocados auditivos de tronco encefálico automático (PEATE-A) presentes bilateralmente. Resultados: obtiveram-se NMR médios de 34.2 dBnNA, 25.4 dBnNA, 19 dBnNA e $17.5 \mathrm{dBnNA}$ para as frequências de $0.5,1,2 \mathrm{e} 4 \mathrm{kHz}$, respectivamente. Os tempos médios de latência da onda $V$ em 70 e $20 \mathrm{dBnNA}$, respectivamente, na frequência de $0.5 \mathrm{kHz}$ foram de $10.75 \mathrm{~ms}$ e 15.2 $\mathrm{ms}$, em $1 \mathrm{kHz}$ de $8.9 \mathrm{~ms}$ e $13.4 \mathrm{~ms}$; de $7.7 \mathrm{~ms}$ e $10.2 \mathrm{~ms}$ em $2 \mathrm{kHz}$, e para $4 \mathrm{kHz}$ foram de $7.3 \mathrm{~ms}$ e 9.4 ms. Conclusão: os valores encontrados podem ser utilizados na prática clínica para orientar o diagnóstico diferencial da perda auditiva, complementando a avaliação auditiva de lactentes.
\end{abstract}

DESCRITORES: Lactente; Testes Auditivos; Vias Auditivas; Potenciais Evocados Auditivos

\section{INTRODUÇÃO}

O diagnóstico audiológico na população pediátrica objetiva, principalmente, o desenvolvimento

(1) Fonoaudióloga colaboradora do Centro "Audição na Criança" da Divisão de Educação e Reabilitação dos Distúrbios da Comunicação da Pontifícia Universidade Católica de São Paulo, CeAC/DERDIC/PUCSP, São Paulo, SP, Brasil; Mestre em Fonoaudiologia pela Pontifícia Universidade Católica de São Paulo; Doutoranda do Programa de Estudos Pós-Graduados em Fonoaudiologia da Pontifícia Universidade Católica de São Paulo.

(2) Fonoaudióloga colaboradora do "Centro Audição na Criança" da Divisão de Educação e Reabilitação dos Distúrbios da Comunicação da Pontifícia Universidade Católica de São Paulo, CeAC/DERDIC/PUCSP, São Paulo, SP, Brasil; Mestre em Fonoaudiologia pela Pontifícia Universidade Católica de São Paulo - PUC-SP; Doutoranda do Programa de Estudos Pós-Graduados em Fonoaudiologia da Pontifícia Universidade Católica de São Paulo.

(3) Fonoaudióloga do "Centro Audição na Criança" da Divisão de Educação e Reabilitação dos Distúrbios da Comunicação da Pontifícia Universidade Católica de São Paulo, CeAC/DERDIC/PUCSP, São Paulo, SP, Brasil; Professora Doutora Titular da Pontifícia Universidade Católica de São Paulo, PUC, São Paulo, SP, Brasil; Professora da Faculdade de Fonoaudiologia e do Programa de Estudos Pós-Graduados em Fonoaudiologia e Centro Audição na Criança, PUC, São Paulo, SP, Brasil; Doutora em Saúde Pública pela Universidade de São Paulo.

Conflito de interesses: inexistente da comunicação e, portanto, há a necessidade de determinação dos limiares auditivos nas freqüências da fala. Em neonatos e lactentes, esses limiares podem apenas ser estimados, uma vez que não é possível sua determinação pela audiometria comportamental de maneira fidedigna ${ }^{1}$.

Nesse contexto, os potenciais evocados auditivos de tronco encefálico (PEATE) são uma ferramenta importante no diagnóstico precoce da perda auditiva. Os PEATE eliciados por estímulos de curta duração, como toneburst, estimulam regiões limitadas da cóclea, originando respostas específicas em frequência. Portanto, possibilitam a estimativa dos limiares auditivos nas diversas frequências e orientam o processo de habilitação auditiva ${ }^{1}$.

A determinação do Nível Mínimo de Resposta (NMR) nos PEATE por frequência específica (PEATE-FE) é realizada pelo registro da onda $V$ e de sua reprodutibilidade, uma vez que esta onda pode ser identificada mesmo em intensidades fracas. Entretanto, muitos autores ressaltam a dificuldade de identificação da onda $V$ nas frequências mais baixas, em decorrência da menor sincronia neural e de uma maior contaminação pelo ruído ${ }^{2}$.

Sabendo-se que a latência da onda $V$ varia com a intensidade e a frequência apresentada, conhecer a latência na qual esta resposta deve ser observada 
é de grande importância para auxiliar em sua identificação, bem como no diagnóstico diferencial do tipo de perda auditiva, ou seja, se condutiva ou sensorioneural ${ }^{3}$.

Estudos enfatizam que os PEATE-FE estimam de maneira eficaz a sensibilidade auditiva por frequência específica em lactentes ${ }^{1,3-9}$ e em adultos ${ }^{3,10} \mathrm{com}$ audição normal ou com perda auditiva.

Investigações prévias ${ }^{11-13}$ sugerem um aumento da latência com a diminuição do nível de intensidade, bem como uma diminuição da latência com o aumento da frequência avaliada.

Este estudo teve o objetivo de determinar 0 NMR e estabelecer a função latência $x$ intensidade da onda $\mathrm{V}$ para o estímulo toneburst nas frequências de 500, 1000, 2000 e $4000 \mathrm{~Hz}$ em lactentes ouvintes e, desta maneira, orientar o diagnóstico de lactentes com perda auditiva.

\section{MÉTODO}

Este é um estudo de caráter experimental e qualitativo.

Todos os participantes passaram na triagem auditiva com Emissões Otoacústicas Evocadas por estímulo Transiente (EOAET) a $73 \mathrm{~dB}$ NPS e nos Potenciais Evocados Auditivos de Tronco Encefálico Automático (PEATE-A) à $35 \mathrm{~dB}$ NPS, por meio do equipamento automático (Accuscreen, marca GN Resound); além de apresentar curva timpanométrica do tipo "A", bilateralmente , afastando alterações de orelha média.

Todos os sujeitos foram avaliados em sono natural.

Os PEATE com o estímulo click, na intensidade de $70 \mathrm{dBnNA}$, foram realizado com o objetivo de verificar a integridade das vias auditivas.

O equipamento utilizado foi o SmartEP da marca Intelligent Hearing Systems, utilizando-se fones de inserção ER 3A. A impedância dos eletrodos foi mantida menor que $3 \Omega$.

Em seguida, deu-se início ao registro dos PEATE-FE por via aérea com estímulo toneburst. Cada criança foi avaliada nas frequências de 0.5 $\mathrm{kHz}, 1 \mathrm{kHz}, 2 \mathrm{kHz}$ e $4 \mathrm{kHz}$, em apenas uma orelha, seguindo os estudos anteriores 5,7,14. Sempre que possível, ambas as orelhas foram avaliadas, em cada um dos sujeitos.

Foi considerado NMR a menor intensidade na qual a onda $V$ pode ser identificada e reproduzida. A estimulação teve início na intensidade de 70 $\mathrm{dBnNA}$ e a pesquisa realizada em passos de $10 \mathrm{~dB}$.

O estímulo foi apresentado na polaridade condensada com taxa de repetição de 39.1/s e janela de análise de $25 \mathrm{~ms}$. Foi utilizado filtro de 30
-1500 na freqüência de $500 \mathrm{~Hz}$ e de 30-3000 nas freqüências de 1 a $4 \mathrm{kHz}$. O estímulo toneburst foi apresentado num envelope Blackman, sem platô, com duração de 8000, 4000, 2000 e $1000 \mu s$ em 0.5, 1 , 2 e $4 \mathrm{kHz}$, respectivamente. Um mínimo de 2000 estímulos foi utilizado para registrar a resposta.

Participaram desta pesquisa, 23 lactentes nascidos a termo, com audição normal e sem indicadores de risco para deficiência auditiva ${ }^{15}$. Destes, 10 lactentes (43\%) eram do sexo masculino e 13 $(57 \%)$ do feminino. Em apenas uma criança foi possível realizar o registro nas duas orelhas, devido ao tempo de exame, e manutenção do sono natural, totalizando desta forma, 24 orelhas. A idade dos lactentes variou de 27 a 105 dias, com média de 70 dias.

O presente estudo foi desenvolvido no Centro Audição na Criança (CeAC) da Divisão de Educação e Reabilitação dos Distúrbios da Comunicação (DERDIC) - PUCSP. O estudo passou pela aprovação do Comitê de Ética da PUCSP, sob protocolo no 114/2008.

Foram realizadas análises estatísticas descritivas dos NMR e latência da onda $V$ para as frequências estudadas (média, mediana, mínimo, máximo e desvio-padrão). Toda a análise descritiva foi feita por sexo.

O teste $t$-Student foi utilizado para comparar as médias das idades de lactentes dos dois sexos.

As distribuições dos NMR nos dois sexos foram comparadas em cada frequência por meio do teste de Mann-Whitney. Para determinar a relação existente entre latência e intensidade em cada frequência, foram estimadas curvas de crescimento.

Nos testes de hipótese foi fixado nível de significância de 0,05.

\section{RESULTADOS}

A média de idade dos lactentes avaliados nesse estudo foi de 70 dias. No sexo feminino, a faixa etária variou de 27 a 90 dias (média de 67,5 dias) e no sexo masculino de 27 a 105 dias (média de 73,1 dias)

Não foi detectada diferença significativa entre as médias das idades dos lactentes entre os sexos $(\mathrm{p}=0,450)$.

\section{Análise descritiva do nível mínimo de resposta (NMR)}

Na Tabela 1 são apresentados os níveis mínimos de resposta (NMR) observados nas frequências de $0.5 \mathrm{kHz}, 1 \mathrm{~Hz}, 2 \mathrm{~Hz}$ e $4 \mathrm{~Hz}$, por sexo.

Não foram encontradas diferenças significantes entre as distribuições do NMR nos dois sexos, para as quatro frequências $(p>0,999 \mathrm{em} 0.5 \mathrm{kHz}$, 
Tabela 1 - Estatísticas descritivas para o NMR nas frequências de 500 HZ, 1000 HZ, $2000 \mathrm{HZ} \mathrm{e} 4000 \mathrm{~Hz}$

\begin{tabular}{cccccccc}
\hline $\begin{array}{c}\text { Frequência } \\
(\mathbf{H z})\end{array}$ & Sexo & $\mathbf{N}$ & Média & $\begin{array}{c}\text { Desvio } \\
\text { padrão }\end{array}$ & Mínimo & Mediana & Máximo \\
\hline \multirow{3}{*}{500} & $\mathrm{~F}$ & 14 & 34,3 & 11,6 & 20 & 30 & 50 \\
& $\mathrm{M}$ & 10 & 34,0 & 10,8 & 20 & 30 & 50 \\
& Total & 24 & 34,2 & 11,0 & 20 & 30 & 50 \\
\hline \multirow{3}{*}{1000} & $\mathrm{~F}$ & 14 & 27,1 & 9,1 & 10 & 30 & 50 \\
& $\mathrm{M}$ & 10 & 23,0 & 6,8 & 10 & 20 & 30 \\
& Total & 24 & 25,4 & 8,3 & 10 & 30 & 50 \\
\hline \multirow{3}{*}{2000} & $\mathrm{~F}$ & 10 & 17,0 & 4,8 & 10 & 20 & 20 \\
& $\mathrm{M}$ & 10 & 21,0 & 5,7 & 10 & 20 & 30 \\
& Total & 20 & 19,0 & 5,5 & 10 & 20 & 30 \\
\hline \multirow{2}{*}{4000} & $\mathrm{~F}$ & 10 & 19,0 & 5,7 & 10 & 20 & 30 \\
& $\mathrm{M}$ & 10 & 16,0 & 7,0 & 10 & 15 & 30 \\
& Total & 20 & 17,5 & 6,4 & 10 & 20 & 30 \\
\hline
\end{tabular}

$\mathrm{p}=0,285$ em $1 \mathrm{~Hz}, \mathrm{p}=0,218$ em $2 \mathrm{kHz}$ e $\mathrm{p}=0,315$ em $4 \mathrm{kHz}$ ).

As médias dos NMR são representadas no diagrama de dispersão da Figura 1. É possível observar que os NMR decrescem de $500 \mathrm{~Hz}$ até
$2000 \mathrm{~Hz}$ e tem respostas similares a partir de 2000 $\mathrm{Hz}$. Observa-se também que o número de sujeitos apresentando NMR em intensidades mais fracas aumenta à medida que aumenta a frequência avaliada.

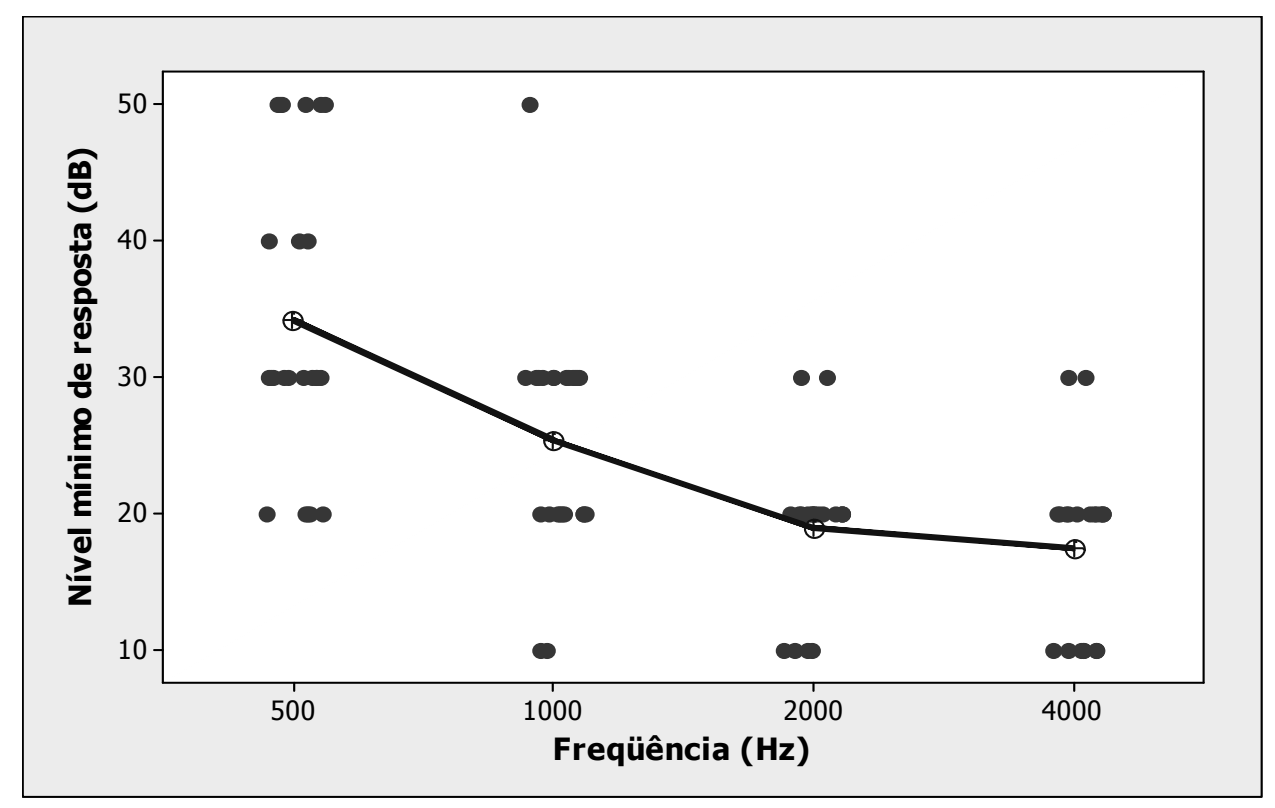

Figura 1 - Diagrama de dispersão do NMR e de frequência em 500 Hz, 1000 Hz, $2000 \mathrm{~Hz}$ e $4000 \mathrm{~Hz}$

Determinação da relação entre Latência e Intensidade nas frequências de $500 \mathrm{~Hz}, 1000 \mathrm{~Hz}$, $2000 \mathrm{~Hz}$ e $4000 \mathrm{~Hz}$

Na Tabela 2 são apresentados os valores observados para a latência da onda $V$ nas frequências de $0.5 \mathrm{kHz}, 1 \mathrm{~Hz}, 2 \mathrm{~Hz}$ e $4 \mathrm{~Hz}$ respectivamente, por sexo.
Não foram observadas diferenças estatisticamente significantes entre os sexos paras as quatro frequências $(p=0,110 \mathrm{em} 0.5 \mathrm{kHz}, p=0,644 \mathrm{em}$ $1 \mathrm{kHz}, p=0,113$ em $2 \mathrm{kHz}$ e $p=0,069$ em $4 \mathrm{kHz}$ ).

Foram construídas curvas de dispersão dos valores de latência nas diversas intensidades que podem ser observadas na figura 2 . 
Tabela 2 - Valores observados de estatísticas descritivas para o tempo de latência (ms) por intensidade (dB) e sexo nas frequências de $500 \mathrm{~Hz}, 1000 \mathrm{~Hz}, 2000 \mathrm{~Hz}$ e $4000 \mathrm{~Hz}$

\begin{tabular}{|c|c|c|c|c|c|c|c|c|c|c|c|c|}
\hline \multirow{2}{*}{$\begin{array}{c}\text { Intensidade } \\
\text { (dBnNA) }\end{array}$} & \multicolumn{3}{|c|}{$500 \mathrm{~Hz}$} & \multicolumn{3}{|c|}{$1000 \mathrm{~Hz}$} & \multicolumn{3}{|c|}{$2000 \mathrm{~Hz}$} & \multicolumn{3}{|c|}{$4000 \mathrm{~Hz}$} \\
\hline & Sexo & $\mathrm{N}$ & Média & Sexo & $\mathbf{N}$ & Média & Sexo & $\mathbf{N}$ & Média & Sexo & $\mathbf{N}$ & Média \\
\hline \multirow{3}{*}{10} & $F$ & --- & ------- & $\mathrm{F}$ & 1 & 14,1 & $\mathrm{~F}$ & 3 & $11,4( \pm 0,9)$ & $\mathrm{F}$ & 2 & $10,1( \pm 1,3)$ \\
\hline & $M$ & --- & ------ & $M$ & 1 & 13,5 & $M$ & 1 & 10,2 & $M$ & 5 & $9,8( \pm 0,8)$ \\
\hline & Total & --- & ------ & Total & 2 & $13,8( \pm 0,4)$ & Total & 4 & $11,1( \pm 1,0)$ & Total & 7 & $9,9( \pm 0,9)$ \\
\hline \multirow{3}{*}{20} & $\mathrm{~F}$ & 3 & $14,6( \pm 0,3)$ & $F$ & 4 & $13,3( \pm 0,5)$ & $F$ & 7 & $9,9( \pm 1,1)$ & $F$ & 7 & $8,8( \pm 0,5)$ \\
\hline & M & 2 & $16,1( \pm 1,0)$ & M & 5 & $13,5( \pm 1,4)$ & $\mathrm{M}$ & 7 & $10,5( \pm 0,7)$ & $\mathrm{M}$ & 4 & $9,4( \pm 0,6)$ \\
\hline & Total & 15 & $15,2( \pm 1,0)$ & Total & 9 & $13,4( \pm 1,0)$ & Total & 14 & $10,2( \pm 1,0)$ & Total & 11 & $9,0( \pm 0,6)$ \\
\hline \multirow{3}{*}{30} & $\mathrm{~F}$ & 9 & $13,8( \pm 1,3)$ & $F$ & 13 & $12,2( \pm 1,1)$ & $F$ & 9 & $9,2( \pm 0,4)$ & $\mathrm{F}$ & 10 & $8,3( \pm 0,5)$ \\
\hline & $\mathrm{M}$ & 6 & $14,4( \pm 1,6)$ & $M$ & 10 & $12,1( \pm 1,0)$ & $\mathrm{M}$ & 10 & $9,6( \pm 0,6)$ & $N$ & 10 & $8,7( \pm 0,5)$ \\
\hline & Total & 15 & $14,0( \pm 1,4)$ & $\mathrm{T}$ & 23 & $12,2( \pm 1,0)$ & Total & 19 & $9,4( \pm 0,6)$ & Total & 20 & $8,5( \pm 0,5)$ \\
\hline \multirow{3}{*}{40} & $\mathrm{~F}$ & 1 & 16,2 & $\mathrm{~F}$ & --- & ------ & $F$ & -- & ------ & $F$ & --- & ------- \\
\hline & $\mathrm{M}$ & 2 & $15,7( \pm 0,3)$ & M & --- & ------ & $\mathrm{M}$ & -- & ------ & $\mathrm{M}$ & --- & ------ \\
\hline & Total & 3 & $15,9( \pm 0,4)$ & Total & --- & ------- & Total & -- & ------- & Total & --- & ------ \\
\hline \multirow{3}{*}{50} & $F$ & 14 & $12,2( \pm 1,5)$ & $F$ & 14 & $9,9( \pm 0,8)$ & $\mathrm{F}$ & 10 & $8,2( \pm 0,4)$ & $\mathrm{F}$ & 10 & $7,7( \pm 0,4)$ \\
\hline & $\mathrm{M}$ & 10 & $13,3( \pm 1,9)$ & M & 10 & $10,4( \pm 0,9)$ & $\mathrm{M}$ & 10 & $8,5( \pm 0,4)$ & $M$ & 10 & $7,9( \pm 0,4)$ \\
\hline & Total & 24 & $12,7( \pm 1,7)$ & Total & 24 & $10,1( \pm 0,9)$ & Total & 20 & $8,3( \pm 0,4)$ & Total & 20 & $7,8( \pm 0,4)$ \\
\hline \multirow{3}{*}{70} & $\mathrm{~F}$ & 14 & $10,2( \pm 1,2)$ & $F$ & 14 & $8,8( \pm 0,8)$ & $F$ & 10 & $7,5( \pm 0,3)$ & $F$ & 10 & $7,2( \pm 0,3)$ \\
\hline & M & 10 & $11,3( \pm 1,5)$ & M & 10 & $9,0( \pm 0,7)$ & $M$ & 10 & $7,8( \pm 0,3)$ & $M$ & 10 & $7,4( \pm 0,4)$ \\
\hline & Total & 24 & $10,7( \pm 1,4)$ & Total & 24 & $8,9( \pm 0,8)$ & Total & 20 & $7,7( \pm 0,3)$ & Total & 20 & $7,3( \pm 0,4)$ \\
\hline
\end{tabular}

Legenda: N: número de sujeitos, ....=não realizado

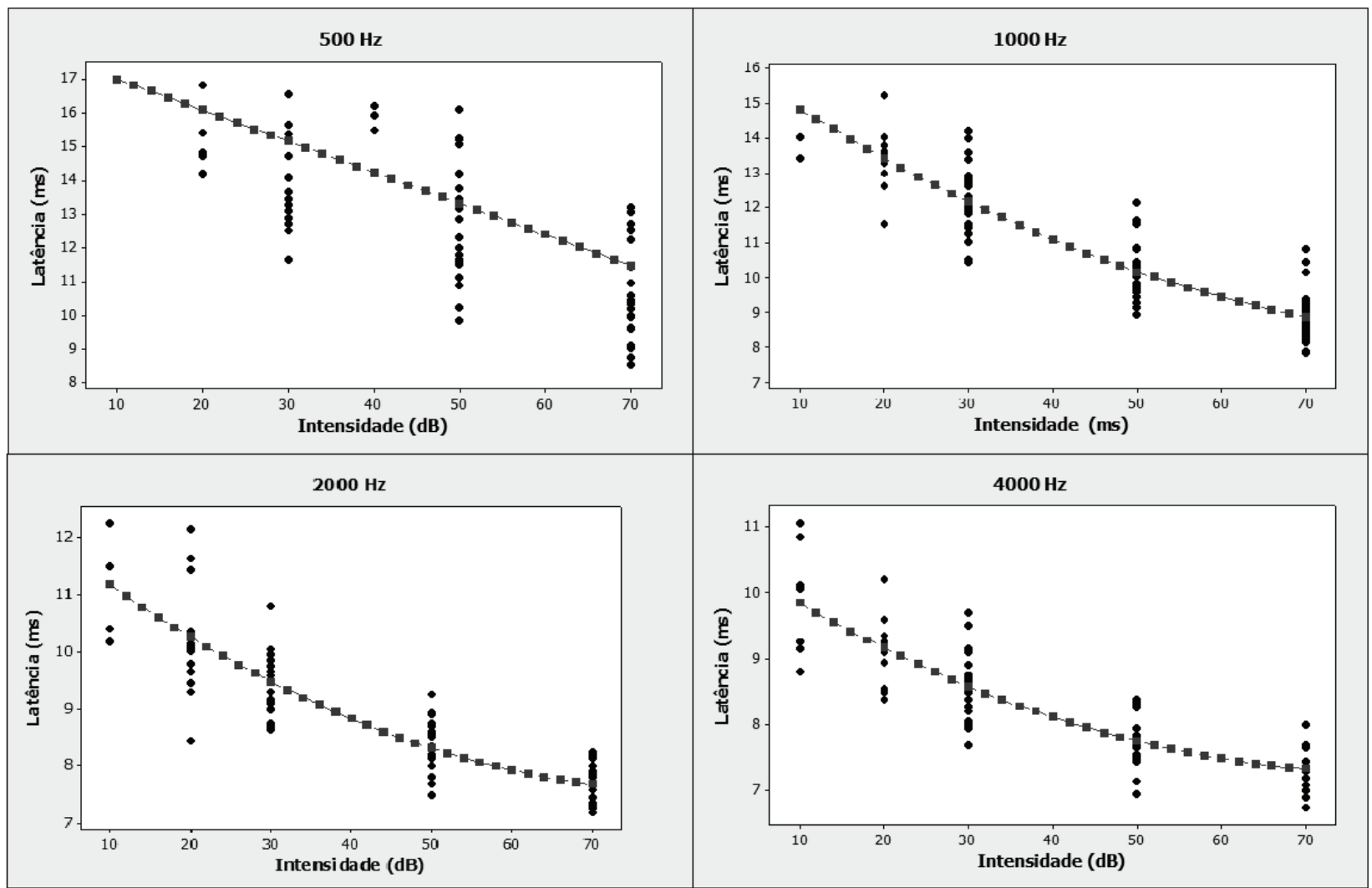

Figura 2 - Diagrama de dispersão da latência em 500 Hz, 1000 Hz, 2000 Hz, 4000 Hz

A Figura 3 demonstra o crescimento da latência em função da intensidade para as quatro frequências estudadas. Observa-se, nesta figura, que a latência apresenta um comportamento inversamente proporcional à intensidade e à frequência; ou seja, a latência diminui quando a intensidade 
aumenta e à medida que a frequência torna-se mais alta.

O tempo médio necessário para a realização dos PEATE-FE nas 4 frequências em uma orelha, considerando as dificuldades relacionadas ao sono e às interrupções do exame quando não havia condições ideais para realizá-lo foi de uma hora e trinta minutos.

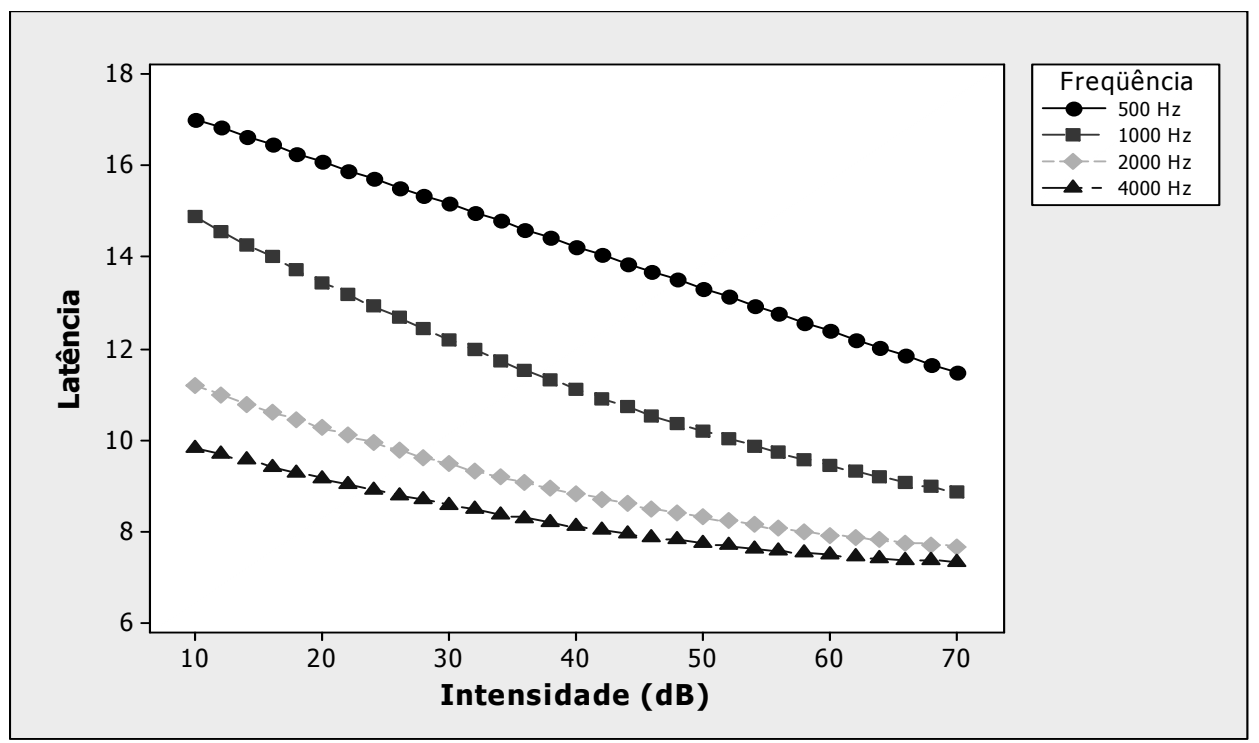

Figura 3 - Curvas de crescimento representativas da função latência $\mathrm{x}$ intensidade obtidas nas frequências de $500 \mathrm{~Hz}, 1000 \mathrm{~Hz}, 2000 \mathrm{~Hz}$ e $4000 \mathrm{~Hz}$

\section{DISCUSSÃO}

Os resultados mostraram NMR em intensidades mais fracas com o aumento da frequência na avaliação pelos PEATE-FE, como já descrito em estudos anteriores ${ }^{1,5,7}$.

A frequência de $500 \mathrm{~Hz}$ apresentou NMR mais elevados em relação às demais frequências estudadas. NMR elevados em $500 \mathrm{~Hz}$ podem ser resultado da dificuldade de identificar a onda $V$ em intensidades muito fracas, decorrente principalmente da morfologia mais arredondada e menor amplitude da onda $V^{8} \mathrm{e}$ da maior contaminação pelo ruído em fracas intensidades ${ }^{2}$. O aumento do número de estímulos por varredura é sugerido para melhorar a relação sinal ruído e possibilitar o registro de NMR em intensidades mais fracas ${ }^{5}$.

A literatura aponta uma variação dos NMR na frequência de $500 \mathrm{~Hz}$ em torno de $16^{5}$ a $40 \mathrm{dBnNA}{ }^{16}$ em lactentes ouvintes. Em metanálise de literatura foi observada uma média de 19,5 dBnNA para o NMR desta frequência em crianças com audição normal $^{8}$.

Em relação às demais frequências, como pode ser observado na Figura 2, o NMR decresceu de $500 \mathrm{~Hz}$ até $2000 \mathrm{~Hz}$ e teve respostas similares a partir de $2000 \mathrm{~Hz}$.
O NMR obtido em $2 \mathrm{kHz}(19,0 \mathrm{~dB})$ está dentro dos $20 \mathrm{dBnNA}$ encontrados em $90 \%$ das crianças avaliadas com os PEATE-FE na literatura especializada ${ }^{4}$. Para a frequência de $4 \mathrm{kHz}$ foram observados no presente estudo valores que corroboram achados anteriormente descritos 4,7,8. Embora, em estudo recente ${ }^{3}$, NMR tenham sido observados na intensidade de $11 \mathrm{~dB}$ NA para RN com até 3 meses de idade.

Os achados do estudo da função latência $X$ intensidade para as quatro frequências foram consistentes com investigações prévias ${ }^{3,12,13}$, as quais sugerem um aumento da latência com a diminuição do nível de intensidade utilizado para desencadear a resposta, bem como uma diminuição da latência com o aumento da frequência avaliada.

Esse comportamento demonstra de forma clara a tonotopia coclear, bem como a especificidade do estímulo utilizado no registro dos PEATE-FE. O aumento da latência nas frequências mais graves reflete a atividade das células ciliadas localizadas na região mais apical da cóclea que, ao serem estimuladas, produzem uma resposta com latência mais longa, já que uma distância maior será percorrida pela onda viajante até atingir as regiões apicais. Em contrapartida, as respostas das células ciliadas da região basal da cóclea apresentam uma latência mais curta, visto que o caminho a ser percorrido pela onda viajante e menor. 
Não foram observadas diferenças estatisticamente significantes na latência da onda $V$, entre os sexos, para as quatro freqüências; corroborando com outros achados em RN a termo ${ }^{12,13}$. Todavia latências ligeiramente melhores para o sexo feminino foram observadas em todas as frequências, na maior parte das intensidades pesquisadas.

$\mathrm{Na}$ avaliação da população pediátrica, o tempo de realização do exame é um fator importante para sua viabilização clínica, principalmente quando se está avaliando crianças pequenas e em sono natural, e quando se precisa considerar a quantidade de pacientes atendidos em um serviço público de saúde auditiva. Neste estudo, o tempo médio para a realização dos PEATE-FE, nas quatro frequências em uma orelha, foi de uma hora e trinta minutos. A presença de artefato no registro- $60 \mathrm{~Hz}$, mesmo com a criança em sono natural e em condições ideais para teste, foi um dos motivos que dificultaram a identificação da onda $\mathrm{V}$, aumentando o tempo de pesquisa, principalmente para as frequências mais baixas.

Apesar das dificuldades encontradas neste estudo para obtenção dos NMR nas quatro frequências estudadas, deve-se considerar que é fundamental, no processo de diagnóstico precoce, a obtenção dos limiares auditivos por freqüência especifica, já nos primeiros meses de vida. Dessa forma, pode-se fornecer informações mais completas e fidedignas que irão auxiliar no processo de seleção e indicação do Aparelho de amplificação sonora individual (AASI).

Além disso, afirmativas anteriores ${ }^{18}$ alertam que ao se utilizar apenas do PEATE com estímulo click ou mesmo o click associado ao PEATE-FE em frequências baixas, há o risco de se diagnosticar como perda auditiva leve às perdas auditivas em rampa.

Estudos $^{19}$ também tem apontado o PEATE-FE como uma ferramenta alternativa no diagnóstico diferencial entre o Espectro da Neuropatia Auditiva e as perdas auditivas cocleares com configuração descendente ${ }^{19}$.Assim, em alguns casos de perda auditiva em frequencias médias e altas, a presença do microfonismo coclear na ausência de resposta para o PEATE-click, pode representar respostas das regiões de frequencias baixas da cóclea. Portanto, também nesses casos, os registros dos PEATE-FE podem fornecer informações importantes para se alcançar um diagnóstico diferencial.

Baseados em todas estas considerações, recomenda-se a obtenção dos PEATE-FE em $500 \mathrm{~Hz}$, para avaliar as frequências baixas e do PEATE-FE em $2000 \mathrm{~Hz}$ para avaliar as frequências altas da cóclea, obtendo-se assim uma idéia da configuração da perda auditiva, e logo em sequência, quando possível, a obtenção das frequências de 1 e $4 \mathrm{kHz}$, como sugerido pelo protocolo de diagnóstico audiológico britânico ${ }^{20}$.

Essa é uma realidade dos centros de diagnóstico audiológico em âmbito internacional e que necessita ser incorporada à realidade dos centros brasileiros ao se considerar a estruturação das novas políticas públicas de saúde auditiva.

\section{CONCLUSÃO}

Diante dos resultados obtidos a partir dos parâmetros de estímulo e registro utilizados nesse estudo, pode-se concluir que os níveis mínimos de resposta em 34,2 dBnNA para a frequência de 500 $\mathrm{Hz}$; de 25,4 dBnNA para a frequência de $1000 \mathrm{~Hz}$ e de 19,0 e $17,5 \mathrm{dBnNA}$ para $2000 \mathrm{~Hz}$ e $4000 \mathrm{~Hz}$ e os valores de latência da onda $\mathrm{V}$ na intensidade de $70 \mathrm{dBnNA}$ em $7,5 \mathrm{~ms}, 8,9 \mathrm{~ms}, 7,7 \mathrm{~ms}$ e $7,3 \mathrm{~ms}$ para as frequências de $500 \mathrm{~Hz}, 1000 \mathrm{~Hz}, 2000 \mathrm{~Hz}$ e $4000 \mathrm{~Hz}$, respectivamente, podem ser considerados normais, podendo ser utilizados como parâmetros na interpretação clínica dos PEATE-FE em lactentes.

\begin{abstract}
Purpose: to determine the ABR thresholds and the latency of $\mathrm{V}$ wave in hearing infants at the frequencies: $0.5,1,2$ and $4 \mathrm{kHz}$. Method: 23 infants with no risk factors concerning hearing loss that had transient otoacoustic emissions (TOAE) and automatic auditory brainstem response (A-ABR), bilaterally present, were evaluated. Results: ABR thresholds were obtained in average, namely: 34.2 $\mathrm{dBHL}, 25.4 \mathrm{dBHL}, 19 \mathrm{dBHL}$ and $17.5 \mathrm{dBHL}$ for frequencies of $0.5,1,2$ and $4 \mathrm{kHz}$, respectively. The mean latency of $\mathrm{V}$ wave at 70 and $20 \mathrm{dBHL}$, respectively, at a $0.5 \mathrm{kHz}$ frequency were $10.75 \mathrm{~ms}$ and $15.2 \mathrm{~ms}, 1 \mathrm{kHz} 8.9 \mathrm{~ms}$ and $13.4 \mathrm{~ms} ; 7.7 \mathrm{~ms}$ and $10.2 \mathrm{~ms}$ at $2 \mathrm{kHz}$, and as for $4 \mathrm{kHz}$ they were 7.3 $\mathrm{ms}$ and $9.4 \mathrm{~ms}$. Conclusions: the found values can be used in clinical practice in order to guide the differential diagnosis of hearing loss, complementing the evaluation as for hearing infants.
\end{abstract}

KEYWORDS: Infant; Hearing Tests; Auditory Pathways; Evoked Potentials, Auditory 


\section{REFERÊNCIAS BIBLIOGRÁFICAS}

1. Gorga MP, Johnson TA, Kaminski JR, Beauchaine KL, Garner CA, Neely ST. Using a combination of click- and tone burst-evoked auditory brain stem response measurements to estimate pure-tone thresholds. Ear Hear. 2006; 27(1):60-74.

2. Sininger YS. The use of auditory brainstem response in screening for hearing loss and audiometric threshold prediction. In: Burkard RF, Dom M and Eggermont JJ . Auditory evoked potentials: basic principles and clinical application. Lippincott Williams \& Wilkins; 2006. p.254-74.

3. Vander Werff KR, Prieve BA, Georgantas LM. Infant Air and Bone Conduction Tone Burst Auditory Brain Stem Responses for Classification of Hearing Loss and the Relationship to Behavioral Thresholds. Ear \& Hear 2009;30:(3)1-19.

4. Stapells DR, Gravel JS, Martin BA. Thresholds for auditory brain stem responses to tones in notched noise from infants and young children with normal hearing or sensorineural hearing loss. Ear Hear. 1995; 16:361-71.

5. Sininger YS, Abdala C, Cone-Wesson B. Auditory threshold sensitivity of neonate as measured by the auditory brainstem response. Hear Res. 1997; (104):27-38.

6. Hurley RM, Hurley A, Berlin C. Development of low-frequency tone burst versus the click auditory brainstem response. J Am Acad Audiol. 2005; 16(2):114 -21.

7. Rance G, Tomlin D, Rickards F. Comparison of auditory steady-state responses and tone-burst auditory brainstem responses in normal babies. Ear Hear. 2006; (27):751-62

8. Stapells DR. Thresholds estimation by toneevoked auditory brainstem response: A literature meta-analysis. J Speech Lang Pathol Audiol. 2000a; 4:74-83.

doi: 10.1590/S1516-18462010005000064

RECEBIDO EM: 16/12/2009

ACEITO EM: 24/03/2010

Endereço para correspondência:

Mabel Gonçalves Almeida

Centro "Audição na Criança" - Divisão de

Educação e Reabilitação dos Distúrbios da

Comunicação PUC/SP

Rua Estado de Israel, 860

São Paulo - SP

CEP: 04022-040

E-mail: mgafono@yahoo.com.br
9. Rodrigues GRI, Almeida MG, Lewis DR. Potenciais evocados auditivos de tronco encefálico por frequência específica e de estado estável na audiologia pediátrica: estudo de caso. Rev Soc Bras Fonoaudiol. 2009;14(4):534-8.

10. Johnson TA, Brown CJ.Threshold Prediction Using the Auditory Steady-State Response and the Tone Burst Auditory BrainStem Response: A WithinSubject Comparison. Ear Hear. 2005;26:559-576.

11. Pinto FR, Matas CG. Comparação entre limiares de audibilidade e eletrofisiológico por estímulo toneburst. Rev Bras Otorrinolaringol. 2007; 73(4):513-22.

12. Ribeiro FGSM. Tone-evoked ABR in full-term and preterm neonates with normal hearing. Int $\mathrm{J}$ Audiol. 2008;47(1):21-9.

13. Sininger YS, Cone-Wesson B, Abdala C. Gender distinctions and lateral asymmetry in the low-level auditory brainstem response of the human neonate. Hear Res. 1998;126:58-66.

14. Van der Reijden C, Mens LH, Snik FM. Frequency-specific objective audiometry: toneevoked brainstem responses and steady-state responses to $40 \mathrm{~Hz}$ and $90 \mathrm{~Hz}$ amplitude modulated stimuli. Int J Audiol. 2006;(45):40-5.

15. JCIH - Joint Committe on Infant Hearing - Year 2007 Position Statement: Principles and guidelines for earl hearing detection and intervention programs. Pediatrics. 2007;120(4):898-921.

16. Hyde ML, Matsumoto N, Alberti PW. The normative basis for click and frequency-specific BERA in high-risk infants. Acta Otolaryngol. 1987;103:602-11.

17. Bhattacharyya N. Auditory Brainstem Response Audiometry. http://emedicine.medscape.com/article/ 836277-overview. Acessado em novembro de 2009. 18. Sininger YS, Martinez A, Eisenberg L, Christensen E, Grimes A, Hu J.Newborn Hearing Screening Speeds Diagnosis and Access to Intervention by $20-25$ Months. J Am Acad Audiol 2009; 20:49-57.

19. Rodrigues GRI, Fichino SN, Lewis DR. Presença de microfonismo coclear no PEATE-clique: diagnóstico diferencial entre espectro da neuropatia auditiva e perdas auditivas cocleares descendentes em crianças. Rev CEFAC [periódico na Internet]. Dez 2009 [acesso em 05 jan 2010]; 11(4):[7]. Disponível em: http://www.scielo.br/scielo.php?pid= S151618462009005000068\&script=sci_arttext 20. BCEHP.(2008). Diagnostic Audiology Protocol... http://www.phsa.ca/NR/rdonlyres/06D79FEBD18 743E991E48C09959F38D8/40115/aDAAG Protocols1.pdf. Acessado em: janeiro de 2009. 